\title{
Erratum
}

Ichthyol Res (2004) 51: 392

DOI 10.1007/s10228-004-0247-1

\section{The karyology of the cyprinid genera Scardinius and Rutilus in southern Europe}

Pier Giorgio Bianco, Gennaro Aprea, Emilio Balletto, Teresa Capriglione, Domenico Fulgione, and Gaetano Odierna

Ichthyol Res (2004) 51(3): 274-278

DOI 10.1007/s10228-004-0221-y

On page 276, in the legend for Figure 2, "S. erythrophthalmus (D)" should read: "S. scardafa (D)," and on page 277, in the legend for Figure 4, "S. erythrophthalmus (G)" should read: "S. scardafa $(\mathbf{G})$." 